\title{
MULTICOLOURED HAMILTON CYCLES
}

\author{
Michael Albert, Alan Frieze and Bruce Reed* \\ Department of Mathematics, Carnegie-Mellon University, \\ Pittsburgh, U.S.A. ${ }^{\dagger}$
}

Submitted: April 25th,1995

Accepted May 9th, 1995

\begin{abstract}
The edges of the complete graph $K_{n}$ are coloured so that no colour appears more than $\lceil c n\rceil$ times, where $c<1 / 32$ is a constant. We show that if $n$ is sufficiently large then there is a Hamiltonian cycle in which each edge is a different colour, thereby proving a 1986 conjecture of Hahn and Thomassen [7]. We prove a similar result for the complete digraph with $c<1 / 64$. We also show, by essentially the same technique, that if $t \geq 3, c<\left(2 t^{2}(1+t)\right)^{-1}$, no colour appears more than $\lceil c n\rceil$ times and $t \mid n$ then the vertices can be partitioned into $n / t$ $t$-sets $K_{1}, K_{2}, \ldots, K_{n / t}$ such that the colours of the $n(t-1) / 2$ edges contained in the $K_{i}$ 's are distinct. The proof technique follows the lines of Erdős and Spencer's [2] modification of the Local Lemma [1].
\end{abstract}

${ }^{*}$ Current address of Bruce Reed: Equipe Combinatoire, CNRS, Université Pierre et Marie Curie, 4 Place Jussieu, Paris, France

${ }^{\dagger}$ Alan Frieze: partially supported by NSF grant CCR-9225008 
The EleCtronic Journal of COMBinatorics 2 (1995), \#R10

\section{Introduction}

Let the edges of the complete graph $K_{n}$ be coloured so that no colour is used more than $k=k(n)$ times. We refer to this as a $k$-bounded colouring. We say that a subset of the edges of $K_{n}$ is multicoloured if each edge is of a different colour. We say that the colouring is H-good if a multi-coloured Hamilton cycle exists i.e., one with a multi-coloured edge-set. Clearly the colouring is $\mathrm{H}$-good if $k=1$ and may not be if $k \geq n / 2$, since then we may only use $n-1$ colours. The main question we address here then is that of how fast can we allow $k$ to grow and still guarantee that a $k$-bounded colouring is $\mathrm{H}$-good.

The problem is mentioned in Erdös, Nestril and Rödl [3]. There they mention it as an Erdös - Stein problem and show that $k$ can be any constant. Hahn and Thomassen [7] were the next people to consider this problem and they showed that $k$ could grow as fast as $n^{1 / 3}$ and conjectured that the growth rate of $k$ could in fact be linear. In unpublished work Rödl and Winkler [9] in 1984 improved this to $n^{1 / 2}$. Frieze and Reed [5] showed that there is an absolute constant $A$ such that if $n$ is sufficiently large and $k$ is at most $\lceil n /(A \ln n)\rceil$ then any $k$-bounded colouring is $\mathrm{H}$-good.

In this paper we remove the $\log n$ factor and prove the conjecture of [7].

Theorem 1 If $n$ is sufficiently large and $k$ is at most $\lceil c n\rceil$, where $c<1 / 32$ then any $k$-bounded colouring of $K_{n}$ is $H$-good.

We can extend this to the directed case.

Theorem 2 If $n$ is sufficiently large and $k$ is at most $\lceil c n\rceil$, where $c<1 / 64$ then any $k$-bounded colouring of the edges of the complete digraph $D K_{n}$ is 
THE ELECTRONiC JOURnAL of COMBinAtorics 2 (1995), \#R10

H-good.

As another wrinkle on this problem, we have

Theorem 3 Suppose the edges of $K_{n}$ are coloured so that the graphs induced by the edges of a single colour all have maximum degree at most cn, where $c<1 / 32$. Then there exists a Hamilton cycle in which each vertex is incident with two edges of a distinct colours.

We prove Theorem's 1, 2 and 3 as corollaries of the following.

Theorem 4 Let $\Gamma$ be a graph whose vertex set is the edge set of $K_{n}$. Suppose that $\Gamma$ has maximum degree bounded above by $c n$, where $c<1 / 32$. Then $K_{n}$ contains a Hamilton cycle $H$ whose edge set is an independent subset in $\Gamma$.

We finally consider multi-coloured sets of cliques of size $t$. More precisely, assume that $t \geq 3, t \mid n$, and let $\mathcal{K}=K_{1}, K_{2}, \ldots, K_{n / t}$ be a partition of $[n]$ into subsets of size $n / t$. We say that $\mathcal{K}$ is multi-coloured if the set of $n(t-1) / 2$ edges which have both endpoints in the same $t$-set is multi-coloured.

Theorem 5 If $n$ is sufficiently large and $k$ is at most $\lceil c n\rceil, c<\left(2 t^{2}(1+t)\right)^{-1}$, then in any $k$-bounded colouring of the edges of $K_{n}$ there is multi-coloured partition $\mathcal{K}$.

\section{Modification of the Lovàsz local lemma}

Let $A_{1}, A_{2}, \ldots, A_{N}$ denote events in some probability space. Using $\bar{A}$ to denote the complement of an event $A$, we are as usual interested in showing that $\operatorname{Pr}\left(\bigcap_{i=1}^{n} \bar{A}_{i}\right)>0$. 
Suppose that for each $i$ there is a partition of $[N] \backslash\{i\}$ into $X_{i}$ and $Y_{i}$. In the usual version of the local lemma, $A_{i}$ will be independent of the the events in $X_{i}$. Here all of the events will be interdependent, but one can still apply the methodology of the usual proof of the local lemma. We should point out here that this idea is not our own, it is already in Erdős and Spencer [2].

We consider one of the terms in the expression

$$
\operatorname{Pr}\left(\bigcap_{i=1}^{N} \bar{A}_{i}\right)=\prod_{i=1}^{N} \operatorname{Pr}\left(\bar{A}_{i} \mid \bigcap_{j=1}^{i-1} \bar{A}_{j}\right)
$$

We want to show that for $1 \leq i \leq N$,

$$
\operatorname{Pr}\left(\bar{A}_{i} \mid \bigcap_{j=1}^{i-1} \bar{A}_{j}\right)>0 .
$$

So, we try to prove by induction on $|S|, S \subseteq[N]$, that for $i \notin S$,

$$
\operatorname{Pr}\left(A_{i} \mid \bigcap_{j \in S} \bar{A}_{j}\right) \leq \alpha,
$$

for some suitable choice of $\alpha$.

Now,

$$
\begin{aligned}
\operatorname{Pr}\left(A_{i} \mid \bigcap_{j \in S} \bar{A}_{j}\right) & =\frac{\operatorname{Pr}\left(A_{i} \cap \bigcap_{k \in S \cap Y_{i}} \bar{A}_{k} \mid \bigcap_{j \in S \cap X_{i}} \bar{A}_{j}\right)}{\operatorname{Pr}\left(\bigcap_{k \in S \cap Y_{i}} \bar{A}_{k} \mid \bigcap_{j \in S \cap X_{i}} \bar{A}_{j}\right)} \\
& \leq \frac{\operatorname{Pr}\left(A_{i} \mid \bigcap_{j \in S \cap X_{i}} \bar{A}_{j}\right)}{\operatorname{Pr}\left(\bigcap_{k \in S \cap Y_{i}} \bar{A}_{k} \mid \bigcap_{j \in S \cap X_{i}} \bar{A}_{j}\right)} \\
& \leq \frac{\operatorname{Pr}\left(A_{i} \mid \bigcap_{j \in S \cap X_{i}} \bar{A}_{j}\right)}{1-\sum_{k \in S \cap Y_{i}} \operatorname{Pr}\left(A_{k} \mid \bigcap_{j \in S \cap X_{i}} \bar{A}_{j}\right)}
\end{aligned}
$$


Let now

$$
\beta=\max \left\{\operatorname{Pr}\left(A_{i} \mid \bigcap_{j \in T} \bar{A}_{j}\right): i \in[N], T \subseteq X_{i}\right\},
$$

and

$$
m=\max \left\{\left|Y_{i}\right|: i \in[N]\right\} .
$$

We will have to prove that given $m, \beta$ we can choose $0 \leq \alpha<1$ such that

$$
\alpha(1-m \alpha) \geq \beta .
$$

Assume that (7) holds. If $S \subseteq X_{i}$ then (5) and (7) will imply

$$
\begin{aligned}
\operatorname{Pr}\left(A_{i} \mid \bigcap_{j \in S} \bar{A}_{j}\right) & \leq \beta \\
& \leq \alpha .
\end{aligned}
$$

On the other hand if $S \nsubseteq X_{i}$ then we can apply the induction hypothesis to $\operatorname{Pr}\left(A_{k} \mid \cap_{j \in S \cap X_{i}} \bar{A}_{j}\right)$ in the numerator of (4) and obtain

$$
\begin{aligned}
\operatorname{Pr}\left(A_{i} \mid \bigcap_{j \in S} \bar{A}_{j}\right) & \leq \frac{\beta}{1-m \alpha} \\
& \leq \alpha
\end{aligned}
$$

by $(7)$.

The base case of the induction, $S=\emptyset$, follows from considering $T=\emptyset$ in (5) and using $\beta \leq \alpha$.

So the proof of (2) rests on proving that (7) holds. This is what we do for Theorem 4 . The proof of Theorem 5 is slightly different, in that we need to partition $A_{1}, A_{2}, \ldots, A_{N}$ into two types of event.

It may be useful to summarise the above discussion as a lemma in case it can be used in other circumstances. 
THE ELECTRONiC Journal of COMBinatorics 2 (1995), \#R10

Lemma 1 Let $A_{1}, A_{2}, \ldots, A_{N}$ denote events in some probability space. Suppose that for each $i$ there is a partition of $[N] \backslash\{i\}$ into $X_{i}$ and $Y_{i}$. Let $m=\max \left\{\left|Y_{i}\right|: i \in[N]\right\}$ and $\beta=\max \left\{\operatorname{Pr}\left(A_{i} \mid \bigcap_{j \in T} \bar{A}_{j}\right): i \in[N], T \subseteq X_{i}\right\}$. If there exists $0 \leq \alpha<1$ such that $\alpha(1-m \alpha) \geq \beta$ then $\operatorname{Pr}\left(\bigcap_{i=1}^{n} \bar{A}_{i}\right)>0$.

\section{$3 \quad$ Hamilton Cycles}

\subsection{Proof of Theorems 1 and 2}

We show here that Theorems 1 and 2 are corollaries of Theorem 4. Assume $n$ is large and $k \leq c n$, and an arbitrary $k$-bounded colouring of $K_{n}$ is given.

To prove Theorem 1 we define $\Gamma$ as follows. Two edges $e, f$ of $K_{n}$ correspond to the endpoints of an edge of $\Gamma$ if and only if they have the same colour. Thus a set of vertices of $\Gamma$ is independent if and only it corresponds to a multicoloured set of edges of $K_{n}$. Clearly the maximum degree of $\Gamma$ is at most $k-1$ and so we can apply Theorem 4 to obtain Theorem 1 .

To prove Theorem 2 we need a slight change in the definition of $\Gamma$. Two edges $e=\left\{e_{0}, e_{1}\right\}, f=\left\{f_{0}, f_{1}\right\}$ of $K_{n}$ define an edge of $\Gamma$ if and only if the colours of the four directed edges $\left(e_{0}, e_{1}\right),\left(e_{1}, e_{0}\right),\left(f_{0}, f_{1}\right),\left(f_{1}, f_{0}\right)$ are not all distinct. Thus a set $S$ of vertices of $\Gamma$ is independent if and only the set of edges obtained by taking, each $e=\left\{e_{0}, e_{1}\right\} \in S$ and replacing it by $\left(e_{0}, e_{1}\right),\left(e_{1}, e_{0}\right)$ (giving $2|S|$ directed edges) is multicoloured. Clearly the maximum degree of $\Gamma$ is at most $2(k-1)$ and so we can apply Theorem 4 to obtain a slight strengthening of Theorem 1 viz. there is a Hamilton cycle and its reversal spanning a multicoloured set of directed edges. 
To prove Theorem 3 we let two edges $e, f$ of $K_{n}$ correspond to the endpoints of an edge of $\Gamma$ if and only if they have the same colour and are incident with a common vertex.

\subsection{Proof of Theorem 4}

Let $H$ be a Hamilton cycle chosen uniformly at random from the set of $(n-$ $1)$ !/2 Hamilton cycles of $K_{n}$. Let $\left\{\left(e_{i}, f_{i}\right): 1 \leq i \leq N\right\}$ be an enumeration of the edges of $\Gamma$. Let

$$
A_{i}=\left\{H: e_{i}, f_{i} \text { are both edges of } H\right\} .
$$

We will prove Theorem 1 by using the argument of Section 2 to show that $\operatorname{Pr}\left(\bigcap_{i=1}^{N} \bar{A}_{i}\right)>0$. We use the notation of that section.

For $1 \leq i \leq N$ let

$$
Y_{i}=\left\{j \neq i:\left(e_{j} \cup f_{j}\right) \cap\left(e_{i} \cup f_{i}\right) \neq \emptyset\right\} .
$$

Thus $j \in Y_{i}$ if in $K_{n}$, one of $e_{j}, f_{j}$ shares a vertex with one of $e_{i}, f_{i}$. Let $X_{i}=[N] \backslash\left(Y_{i} \cup\{i\}\right)$. Clearly, $\left|Y_{i}\right| \leq 4 c n^{2}$ and so

$$
m \leq 4 c n^{2}
$$

We will show that

$$
\beta \leq \frac{2}{n^{2}-15 n+56}
$$

and Theorem 1 follows on choosing

$$
\alpha=\frac{1}{8 c}(1-\sqrt{1-(32+\epsilon) c}) n^{-2}
$$

and checking that (7) holds for $\epsilon>0$ sufficiently small and $n$ sufficiently large. 
$[$ Now $m \alpha \leq(1-\sqrt{1-(32+\epsilon)}) / 2$ and so $1-m \alpha \geq(1+\sqrt{1-(32+\epsilon)}) / 2$. Thus $\alpha(1-m \alpha)>(2+\epsilon / 16) n^{-2}$.]

Equation (8) follows from the following Lemma:

Lemma 2 Let $e, f$ be edges of $K_{n}$ and $X \subseteq E\left(K_{n}\right)$ be such that no edge in $X$ shares an endpoint with either $e$ or $f$. Then we can find, for each Hamilton cycle $C$ containing both $e$ and $f$ and no edges of $X$, a set $S(C)$ of $(n-6)(n-9) / 2$ Hamilton cycles containing neither $e, f$ or any edge in $X$, in such a way that if $C \neq C^{\prime}$ then $S(C) \cap S\left(C^{\prime}\right)=\emptyset$.

Proof Let $e_{0}, e_{1}$ and $f_{0}, f_{1}$ be the endpoints of $e$ and $f$ respectively, chosen so that $e_{0}$ has the smallest index of $e_{0}, e_{1}, f_{0}, f_{1}$. Let

$$
C=e_{0}, e_{1} \longrightarrow f_{0}, f_{1} \longrightarrow e_{o}
$$

[It is possible that $e_{1}=f_{0}$ here.]

Consider two disjoint edges $x=\left(x_{0}, x_{1}\right), y=\left(y_{0}, y_{1}\right)$ of $C$ sharing no endpoint with $e$ or $f$. There are at least $(n-6)(n-9) / 2$ choices for $x, y$. There are now two possibilities:

$$
C=e_{0}, e_{1} \longrightarrow x_{0}, x_{1} \longrightarrow f_{0}, f_{1} \longrightarrow y_{0}, y_{1} \longrightarrow e_{o}
$$

or

$$
C=e_{0}, e_{1} \longrightarrow f_{0}, f_{1} \longrightarrow x_{0}, x_{1} \longrightarrow y_{0}, y_{1} \longrightarrow e_{o}
$$

In the first case define:

$$
\hat{C}_{x, y}=e_{0}, x_{0} \longrightarrow e_{1}, y_{0} \longrightarrow f_{1}, x_{1} \longrightarrow f_{0}, y_{1} \longrightarrow e_{o}
$$

In the second case define: 
THE ELECTRONiC Journal of COMBinatorics 2 (1995), \#R10

$$
\hat{C}_{x, y}=e_{0}, x_{1} \longrightarrow y_{0}, f_{1} \longrightarrow x_{0}, e_{1} \longrightarrow f_{0}, y_{1} \longrightarrow e_{o}
$$

In both cases we delete the edges $e, f, x, y$ from $C$ and add edges that are incident with one of $e_{0}, e_{1}, f_{0}, f_{1}$, so that $\hat{C}_{x, y}$ does not contain an edge of $X$.

It is important to realise that in both cases the procedure is reversible in that $C$ can be reconstructed from $\hat{C}_{x, y}$. We can recognise which case we are in from the relative order of the $e$ 's and f's and then identify the $x$ 's and $y$ 's from their positions.

Thus taking $S(C)=\left\{\hat{C}_{x, y}: x, y\right.$ as above $\}$, we obtain $|S(C)| \geq(n-6)(n-$ $9) / 2$ and $S(C) \cap S\left(C^{\prime}\right)=\emptyset$ for $C \neq C^{\prime}$.

To prove (8) we apply Lemma 2 with $i \in[N],\{e, f\}=\left\{e_{i}, f_{i}\right\}$ and $X \subseteq X_{i}$. Let $\mathcal{C}$ denote the set of Hamilton cycles containing $e_{i}$ and $f_{i}$. Then

$$
\begin{aligned}
\operatorname{Pr}\left(A_{i} \mid \bigcap_{j \in X} \bar{A}_{j}\right) & =\sum_{C \in \mathcal{C}} \operatorname{Pr}\left(H=C \mid \bigcap_{j \in X} \bar{A}_{j}\right) \\
& \leq \frac{2}{n^{2}-15 n+56} \sum_{C \in \mathcal{C}} \operatorname{Pr}\left(H \in\{C\} \cup S(C) \mid \bigcap_{j \in X} \bar{A}_{j}\right) \\
& \leq \frac{2}{n^{2}-15 n+56} .
\end{aligned}
$$

This completes the proof of Theorem 4.

\section{Partition into cliques}

Assume $n$ is large and $k \leq c n, c<\left(2 t^{2}(1+t)\right)^{-1}$, and an arbitrary $k$ bounded colouring of $K_{n}$ is given. Let $\left\{\left(S_{1}, T_{1}\right),\left(S_{2}, T_{2}\right), \ldots,\left(S_{N}, T_{N}\right)\right\}$ be an enumeration of the pairs of $t$-subsets of $[n]$ such that for each $1 \leq i \leq N$ either (a) $S_{i}=T_{i}$ and $S_{i}$ contains a pair of edges $e, f$ of the same colour, or 
(b) $S_{i} \cap T_{i}=\emptyset$ and there are edges $e, f$ of the same colour, $e \subseteq S_{i}, f \subseteq T_{i}$. In either case we say that $\left(S_{i}, T_{i}\right)$ contains $e, f$.

Let $I_{a}=\left\{i \in[N]: S_{i}=T_{i}\right\}$ and $I_{b}=[N] \backslash I_{a}$. Now let $\mathcal{K}$ be chosen randomly from the set of possible partitions and define the events

$$
A_{i}=\left\{S_{i} \text { and } T_{i} \text { are both members of } \mathcal{K}\right\}
$$

Once again, we prove that $\operatorname{Pr}\left(\bigcap_{i=1}^{n} \bar{A}_{i}\right)>0$.

We now define $Y_{i}=\{j \neq i$ : the following three conditions hold:

1. $\max \left\{\left|S_{j} \cap S_{i}\right|,\left|S_{j} \cap T_{i}\right| \geq t-1\right.$.

2. $\max \left\{\left|T_{j} \cap S_{i}\right|,\left|T_{j} \cap T_{i}\right| \geq t-1\right.$.

3. $\left(S_{j}, T_{j}\right)$ contains a pair of identically coloured edges $e, f$ which are not contained in $\left.\left(S_{i}, T_{i}\right)\right\}$.

Naturally, $X_{i}=[N] \backslash\left(Y_{i} \cup\{i\}\right)$.

We elaborate the argument of Section 2. We prove the existence of $0<$ $\alpha_{a}, \alpha_{b}<1$ such that if $S \subseteq[N]$ and $i \in I_{x} \backslash S$, where $x=a$ or $b$, then

$$
\operatorname{Pr}\left(A_{i} \mid \bigcap_{j \in S} \bar{A}_{j}\right) \leq \alpha_{x}
$$

To do this, we define, for $x=a$ or $b$,

$$
\beta_{x}=\max \left\{\operatorname{Pr}\left(A_{i} \mid \bigcap_{j \in T} \bar{A}_{j}\right): i \in I_{x}, T \subseteq X_{i}\right\}
$$

and

$$
m_{x}=\max \left\{\left|Y_{i}\right|: i \in I_{x}\right\}
$$


The EleCtronic Journal of COMBinatorics 2 (1995), \#R10

We will then, in analogy with (7), only need to show that for $x=a$ or $b$,

$$
\alpha_{x}\left(1-m_{a} \alpha_{a}-m_{b} \alpha_{b}\right) \geq \beta_{x}
$$

Consider first the case where $i \in I_{a}$. Then $j \in X_{i}$ implies $j \in I_{a}$.

$\left[\left|S_{j} \cap T_{j}\right| \geq\left|S_{j} \cap S_{i} \cap T_{j} \cap S_{i}\right| \geq\left|S_{j} \cap S_{i}\right|+\left|T_{j} \cap S_{i}\right|-\left|S_{i}\right| \geq 2(t-1)-t>0.\right]$

Now

$$
m_{a} \leq t\left(\begin{array}{c}
t-1 \\
2
\end{array}\right) k .
$$

Explanation: We choose $S_{j}$ by (i) choosing $x \in S_{i}$, (ii) $e=\left\{x_{1}, x_{2}\right\} \subseteq$ $S_{i} \backslash\{x\}$ and then $y \notin S_{i}$ such that the colour of $e$ is the same as that of $\left\{x_{1}, y\right\}$ or $\left\{x_{2}, y\right\}$. We then take $S_{j}=\left(S_{i} \backslash\{x\}\right) \cup\{y\}$.

We argue next that

$$
\beta_{a} \leq \frac{1}{t(n-t+1)}
$$

Explanation: Given $\mathcal{K} \in A_{i} \cap \bigcap_{j \in T} \bar{A}_{j}, T \subseteq X_{i}$, we can obtain $t(n-t)$ distinct partitions which are in $\bar{A}_{i} \cap \bigcap_{j \in T} \bar{A}_{j}$ as follows: Choose $x \in S_{i}$ and $y \in S$, where $S$ is another $t$-set of $\mathcal{K}$. Replace $S_{i}$ by $\left(S_{i} \cup\{y\}\right) \backslash\{x\}$ and $S$ by $(S \cup\{x\}) \backslash\{y\}$ to obtain $\mathcal{K}^{\prime}$. Note that given $\mathcal{K}^{\prime}$ we can re-construct $\mathcal{K}$ : $x$ is the unique element of $S_{i}$ which is in a set with elements not in $S_{i}$ and $y \notin S_{i}$ is the unique such element which in a set with $t-1$ members of $S_{i}$.

Now let us consider the case $i \in I_{b}$. Now $j \in X_{i}$ implies that $j \in I_{b}$. Also,

$$
m_{b} \leq t^{2}\left(\begin{array}{c}
t-1 \\
2
\end{array}\right)(t-1)(n-t) k .
$$

Explanation: We choose $S_{j}, T_{j}$ by (i) choosing $x \in S_{i}, y \in T_{i}$, (ii) $e=$ $\left\{x_{1}, x_{2}\right\} \subseteq S_{i} \backslash\{x\}$, (iii) $z \notin S_{i}$, (iv) $w \in T_{i} \backslash\{y\}$, (v) $v \in[n]$ such that the colour of $\{v, w\}$ is the same as that of $e$. Then take $S_{j}=\left(S_{i} \backslash\{x\}\right) \cup\{z\}$ 
and $T_{j}=\left(T_{i} \backslash\{y\}\right) \cup\{w\}$. There are some restrictions on the choices of $x, y, z, v, w$ which are ignored for the purposes of getting an upper bound.

Finally,

$$
\beta_{b} \leq \frac{1}{t^{2}(n-2 t)(n-3 t)+1} .
$$

Explanation: Given $\mathcal{K} \in A_{i} \cap \bigcap_{j \in T} \bar{A}_{j}, T \subseteq X_{i}$, we can obtain $t^{2}(n-2 t)(n-$ $3 t)$ distinct partitions which are in $\bar{A}_{i} \cap \bigcap_{j \in T} \bar{A}_{j}$ as follows: Choose $x \in S_{i}$ and $y \in T_{i}$. Then choose $x^{\prime}, y^{\prime} \notin S_{i} \cup T_{i}$ in distinct subsets $S, S^{\prime}$ of $\mathcal{K}$. Replace $S_{i}$ by $\left(S_{i} \cup\left\{x^{\prime}\right\}\right) \backslash\{x\}, T_{i}$ by $\left(T_{i} \cup\left\{y^{\prime}\right\}\right) \backslash\{y\}, S$ by $(S \cup\{x\}) \backslash\left\{x^{\prime}\right\}$ and $T$ by $(T \cup\{y\}) \backslash\left\{y^{\prime}\right\}$. to obtain $\mathcal{K}^{\prime}$. Note once again, that given $\mathcal{K}^{\prime}$ we can re-construct $\mathcal{K}$.

With these values for $m_{a}, m_{b}, \beta_{a}, \beta_{b}$ we can enforce (9) by choosing

$$
\alpha_{a}=2 / t \text { and } \alpha_{b}=2 / t^{2}
$$

This completes the proof of Theorem 5 .

\section{References}

[1] N.Alon and J.H.Spencer, The probabilistic method, John Wiley and Sons, New York, 1992.

[2] P.Erdős and J.Spencer, Lopsided Lovász local lemma and Latin transversals, Discrete Applied Mathematics 30 (1990) 151-154.

[3] P.Erdös, J.Nesetril and V.Rödl, Some problems related to partitions of edges of a graph in Graphs and other Combinatorial topics, Teubner, Leipzig (1983) 54-63. 
[4] T.I.Fenner and A.M.Frieze, On the existence of polychromatic sets of edges in graphs and digraphs, Progress in Graph Theory, Edited by J.A. Bondy and U.S.R. Murty, Academic Press, 219-232.

[5] A.M.Frieze and B.A.Reed, Polychromatic Hamilton cycles, Discrete Mathematics 118, (1993) 69-74.

[6] W.Höeffding, Probability inequalities for sums of bounded random variables, Journal of the American Statistical Association 58 (1963) 13-30.

[7] G.Hahn and C.Thomassen, Path and cycle sub-Ramsey numbers and an edge-colouring conjecture, Discrete Mathematics 62 (1986) 29-33.

[8] L.Pósa,Hamilton circuits in random graphs,Discrete Mathematics 14 (1976) 359-64.

[9] P.Winkler, Private Communication. 